\title{
Programa de gestión y dirección educativa basada en técnicas de relaciones públicas, su eficiencia en la motivación del personal docente del Instituto Computron del distrito de Los Olivos - Lima, 2013
}

\author{
K ovács Halay, István János ${ }^{1}$; A cuña Casas, R aúl ${ }^{2}$ \\ 1,2Universidad Peruana Unión (UPeU), Perú
}

Recibido el 1 de agosto de 2014 - A ceptado el 28 de octubre de 2014

\begin{abstract}
Resumen
La presente investigación aplicó un programa de gestión educativa con las técnicas de relaciones públicas, principalmente la de administración de la controversia pública para fortalecer eficientemente la motivación del personal docente del Instituto de Educación Superior COM PUTRON aportando al campo de la gestión educativa una nueva teoría en donde se demuestra que puede ser más eficiente si es que los directivos de una entidad educativa aplican conocimientos y programas de relaciones públicas. Para ello se aplicó el programa en dicho instituto en el año 2013 en sus sedes de los distritos de Los Olivos y San J uan de Lurigancho, respectivamente para conocer el nivel de motivación e identificación del personal docente con dicha institución, lo cual implicó conocer el concepto que tienen sobre el instituto COM PUTRON. EI primero fue nuestro grupo experimental con 33 docentes, al cual se aplicó este programa de gestión educativa con técnicas de relaciones públicas durante seis meses con seis sesiones de tres horas cada una, totalizando el experimento dieciocho horas teóricas y prácticas. Luego de ello se les aplicó el mismo test como postexperimento, demostrando que hubo cambios positivos de manera eficiente en cuanto al nivel de motivación del personal docente respecto al instituto. En tanto, la segunda población fue el grupo control con 35 docentes a quienes no se les aplicó este programa, pero también se les sometió al postest. Luego, se compararon ambos resultados, obteniendo las diferencias respectivas que demostraron que este programa de gestión educativa aplicado con las técnicas de relaciones públicas es eficiente mejorando significativamente el nivel de motivación del docente. Por ello, esta investigación tiene trascendencia social porque se puede aplicar en cualquier entidad educativa dentro y fuera del país y al mismo tiempo resuelve el problema de la falta de motivación docente en la participación de actividades que organiza la institución educativa en sí.
\end{abstract}

Palabras claves: Relaciones públicas, programa de gestión educativa, motivación, eficiencia, personal docente, instituto de educación superior.

Abstract

This research applied a program of educational management techniques Public Relations $M$ anagement mainly public controversy to efficiently increase the motivation of teachers of the Institute of Higher Education COM PUTRON contributing to the field of educational management where a new theory shows that it can be more efficient if the directors of an educational institution and apply knowledge of public relations programs. This program was implemented in the institute in 2013 at its headquarters in the districts of Los Olivos and San J uan de L urigancho, respectively for the motivation and identification with the institution staff, which meant knowing the concept they have on the COM PUTRON institute. The first was our pilot group of 33 teachers, to which this program was applied educational management with Public Relations techniques for six months in six sessions of three hours each one, totaling eighteen hours the experiment and theoretical practices. After that we applied the same test as a post experiment, showing that there were positive changes efficiently in the level of motivation of teachers regarding the school. M eanwhile, the second population was the control with 35 teachers who have not applied this program, but are also subjected to post-test group. Then, both results were compared, obtaining the respective differences showed that this educational program implemented management techniques Public Relations is efficient to significantly improve the level of motivation of teachers. Therefore, this research has social significance because it can be applied in any educational institution within and outside the country and also solves the problem of the lack of teacher motivation in participation activities organized by the school itself.

K eywords: Public Relations, Educational program management, motivation, efficiency, faculty, Institute of Higher Education.

\section{Introducción}

La presente investigación titulada "Programa de gestión y dirección educativa basada en técnicas de Relaciones Públicas, su eficiencia en la motivación del personal docente del Instituto Computron de Los Olivos Lima, 2013, es producto de la preocupación por parte del investigador de resolver un problema común en las instituciones educativas de nuestro país como es la falta de un nivel adecuado de motivación del personal docente respecto a la institución educativa para la cual labora. D e 
allí que el título de la presente investigación resalte el punto de mejorar dicha motivación como un indicador de éxito en la gestión que desarrollen los directivos y promotores de la entidad educativa en sí.

Este problema hizo que en nuestro contexto social se haya hecho costumbre que gran parte del personal docente no participe por convicción de las actividades sociales 0 académicas programadas por la directiva de la institución educativa, sino que lo realicen por presión 0 coacción utilizando la continuidad laboral como medio de ejercer presión por parte de los directivos. Eso lejos de generar sentido de pertenencia, genera más bien un distanciamiento entre docentes y directivos y la consiguiente falta de identificación con la entidad educativa para la cual laboran quienes imparten el servicio educativo mediante la enseñanza. La coacción no genera ánimos positivos para colaborar y participar, sino sumisión, la cual es un sentimiento de obediencia pero sin un sincero ánimo por cooperación que a largo plazo acumula sentimientos de rechazo hacia los directivos quienes para asegurar dicha participación cometen el error de obligar lejos de motivar a que su personal docente sea partícipe con entusiasmo e identificación plena con la entidad para la cual laboran. A ello se refiere Pereyra $(2006, p .71)$ cuando afirma que "es importante la autorrealización del cliente en el ejercicio de la elección y la decisión personal en un ámbito de libertad y responsabilidad en donde la relación interpersonal es importante". Gran parte de este problema se encuentra en la falta de una comunicación constante y democrática que deben fomentar quienes tienen la responsabilidad de gestionar a la entidad educativa, por lo que dicha gestión sin comunicación está irremediablemente condenada al fracaso y la consiguiente baja motivación para colaborar y participar en las actividades que los directivos propongan, sea para el bien de los mismos docentes o para los educandos. En lo que se refiere específicamente al Instituto de Educación Superior Computron, este problema no es ajeno, por lo que en la presente investigación se buscó mejorar el aspecto de la comunicación mediante la aplicación de las técnicas de Relaciones Públicas fomentando, entre otros aspectos, el desarrollo de la administración de la controversia pública, la cual a su vez, resultó ser la técnica de Relaciones Públicas más aceptada por parte de los docentes.

Es de conocimiento común que en un grupo humano existan divergencias de ideas y que, si bien es cierto dicho equipo de trabajo apunta a metas y objetivos comunes, cada integrante posee un punto de vista personal respecto a la forma cómo deben de cumplirse con dichas objetivos. Aquí es donde surgen las controversias, que si no son debidamente administradas por los encargados de la gestión de la entidad educativa, pueden degenerar en conflictos que a su vez dañan la buena imagen y el prestigio que busca la institución educativa en la comunidad para poder crecer y lograr la aceptación por parte de estudiantes y los docentes mismos que requiere para su desarrollo.

Tomando en cuenta que la gestión educativa que motive a los docentes a identificarse mejor con la misma institución educativa parte del conocimiento de las necesidades y expectativas del personal docente, es preciso conocer dichas necesidades mediante una gestión que fomente el desarrollo de canales de comunicación que permita a la administración saber de antemano con quiénes están trabajando en la institución y al mismo tiempo saber escuchar en forma oportuna lo que el personal docente tiene que decir sobre cómo se gestiona en sí a la institución educativa. Este aspecto lo hace notar Ríos (1996, p. 37) cuando afirma que "de nada servirá tratar de convencer a la sociedad sobre el prestigio y la buena imagen de una organización mediante costosas campañas publicitarias en los medios de comunicación si es que no está fundamentada en la identificación plena del personal interno de dichas organizaciones, ya que en la sociedad actual los públicos tienden a ser más cuidadosos al momento de dar su confianza a una organización y toman muy en cuenta los comentarios de la propia gente que labora 0 pertenece a dichas organizaciones. Por eso es que cualquier gestión debe preocuparse más por el sentir de su propio personal en primer lugar antes que en buscar generar una imagen externa basándose solamente en apariencias que los testimonios de quienes forman parte de la organización puedan desvirtuarlos con facilidad". Por ello es necesario conocer a cada docente en forma individual y hacerlo sentir parte de la institución, no solamente como un personal de apoyo, sino como persona integrante de la familia o comunidad educativa que tiene mucho que aportar en bien de la institución. Con esta apreciación, coincide plenamente Knight (2009, p.176) cuando afirma que "la mejor vía para los educadores y los sistemas educativos consiste en examinar individualmente sus propias creencias básicas en términos de la realidad, la verdad y el mérito, y luego estructurar conscientemente una filosofía personal de la educación sobre esa plataforma". Esta afirmación eminentemente hace referencia a la forma de gestionar 
una institución educativa mediante el conocimiento pleno de la realidad de cada docente.

Buscando la solución para esta problemática es que desde julio hasta diciembre de 2013 se desarrolló el programa de gestión educativa con la aplicación de las técnicas de relaciones públicas en el personal docente y los directivos del Instituto de Educación Superior Computron de la sede del distrito de Los Olivos, partiendo de la premisa que las relaciones públicas al fomentar la buena comunicación entre los integrantes de una organización pueden ayudar a la generación de una motivación positiva en el personal docente.

Por esta razón es que lo innovador de esta investigación consiste en aplicar las técnicas de relaciones públicas, principalmente la técnica de la administración de la controversia pública que consiste en equilibrar intereses y objetivos entre los docentes y la directiva para lograr acuerdos entre ambas partes y así trabajar en forma conjunta sin que ninguna de las partes sienta que ha perdido en beneficio de la contraparte. Esto posibilita que dichos programas de gestión educativa tengan una mejor acogida, y en consecuencia, logren la motivación e identificación necesaria de los integrantes de la institución en sí. Por estas razones, los beneficios y aportes de esta investigación son múltiples.

Para ello se aplicó un programa de gestión educativa con la aplicación de las técnicas de relaciones públicas, el cual fue realizado desde julio hasta diciembre de 2013. Consistió en aplicar un pretest entre el personal docente de dos sedes del Instituto de Educación Superior Computron: del distrito de Los Olivos y de San Juan de L urigancho, ambos en la ciudad de L ima para conocer el nivel de motivación de los docentes entre ambas sedes, además de conocer el concepto que tienen sobre la institución en mención. El primer grupo de 33 docentes fue nuestro grupo experimental, al cual se aplicó el programa de gestión educativa con las técnicas de relaciones públicas en conjunto con los directivos desde julio hasta diciembre de 2013 consistente en 6 sesiones de 3 horas cada una durante las reuniones mensuales de coordinación académica que convoca la gerencia general con los docentes, mediante el desarrollo de dinámicas grupales, administración de la controversia pública y trabajos de asesoría personalizada a la gerencia general para reorientar su gestión educativa. Luego de ello a los docentes se les aplicó el mismo test como postest, demostrando que hubo cambios positivos en cuanto al nivel de motivación y que al mismo tiempo existió una mejoría en la percepción docente sobre la gestión educativa que desarrolla la gerencia general del Instituto de Educación Superior Computron. En tanto, la segunda población fue el grupo control conformado por otros 35 docentes a quienes no se les aplicó el programa de gestión educativo con las técnicas de relaciones públicas, pero que también se les sometió al postest. Luego, se compararon ambos resultados, obteniendo las diferencias respectivas que nos demostraron que un programa de gestión educativa con la aplicación de las técnicas de relaciones públicas son eficientes en la mejoría de la motivación docente en instituciones educativas como el Instituto de Educación Superior Computron, hecho que puede ser generalizado y aplicado a diversas instituciones educativas tanto en Lima como en nuestro país y en el exterior.

En conclusión, la presente investigación ayuda a resolver el gran problema del bajo nivel de motivación del personal docente hacia diversas instituciones educativas producto de una gestión educativa que es ineficiente en cuanto al establecimiento de políticas de comunicación que permitan sintonizar los intereses de todos los miembros de la comunidad educativa.

\section{Método de la investigación}

La secuencia lógica de la presente tesis estuvo basada en el desarrollo de un trabajo de campo mediante una investigación cuasi experimental, la cual constó de las siguientes partes:

Establecer y formular las hipótesis a demostrar en esta investigación para conocer si eran válidas y aplicables en la realidad, para convertirse en teorías. Las hipótesis con las que se ha trabajado fueron las siguientes:

\section{Hipótesis general:}

EI programa de gestión y dirección basada en técnicas de relaciones públicas es eficiente en el desarrollo de la motivación de los docentes del Instituto Computron del distrito de L os Olivos - Lima, año 2013.

\section{Hipótesis específicas}

H1:El programa de gestión y dirección educativa basada en técnicas de relaciones públicas es eficiente en el desarrollo de la realización personal de los docentes del Instituto Computron del distrito de Los Olivos Lima, año 2013. 
H2EI programa de gestión y dirección educativa basada en técnicas de relaciones públicas es eficiente en el reconocimiento de los aportes de los docentes del Instituto Computron del distrito de Los Olivos Lima, año 2013.

H3:EI programa de gestión y dirección educativa basada en técnicas de relaciones públicas es eficiente en el desarrollo de la responsabilidad de los docentes del Instituto Computron del distrito de Los Olivos Lima, año 2013.

H4:EI programa de gestión y dirección educativa basada en técnicas de relaciones públicas es eficiente en la adecuación a las condiciones de trabajo de los docentes del Instituto Computron del distrito de LOS Olivos - Lima, año 2013.

Establecidas las hipótesis de estudios, se procedió con los siguientes pasos:

Selección de dos grupos de investigación: El primero, el grupo experimental que estuvo constituido por 33 docentes del Instituto de Educación Superior Computron del distrito de Los Olivos, a quienes se les sometió al experimento consistente en un programa de gestión educativa con las técnicas de relaciones públicas. Para la comparación respectiva, se designó a un segundo grupo denominado como el de control, conformado por 35 docentes pertenecientes a la misma institución educativa de la sede del distrito de San Juan de Lurigancho, a quienes e les observó pero no se les sometió al programa de gestión educativa.

El tiempo del desarrollo de la investigación tuvo una duración de 6 meses, de julio a diciembre de 2013, el cual constó de 6 sesiones de 3 horas cada una, totalizando 18 horas del desarrollo del experimento para el grupo de 33 docentes de la sede del Instituto de Educación Superior Computron de la sede del distrito de Los Olivos. Durante el mismo tiempo se hizo una observación al grupo control.

Paralelamente al desarrollo del experimento con los docentes, también se asesoró de manera permanente a la dirección del instituto representado por el gerente general, el Señor Richar Bacón Terrones para la optimización del programa de gestión educativa con las técnicas de relaciones públicas hacia los docentes.
Antes de iniciar la experimentación, se diseñó el instrumento de investigación consistente en una encuesta con estaca tipo Likert, la cual fue validada por 5 doctores expertos en educación, la misma que fue el test que sirvió para medir el nivel de motivación docente antes y después de la experimentación. El instrumento pretest y postest consta de 16 ítemes divididos en 4 áreas de observación: ideal de realización personal, nivel de reconocimiento de aportes, nivel del desarrollo de la responsabilidad docente y nivel de adecuación a las condiciones de trabajo. Cada pregunta era respondida de acuerdo a la frecuencia de ocurrencia en la escala del 0 al 5 , siendo las respuestas: Nunca (0 puntos), rara vez (1 punto), a veces ( 2 puntos), regularmente (3 puntos), casi siempre (4 puntos) y siempre (5 puntos). De estas preguntas los puntajes para medir globalmente el nivel de motivación de los docentes era de acuerdo a las siguientes escalas por intervalos: 0-16 deficiente, 17-32 bajo, 33-48 moderado (promedio), 49-64 alto y 65-80 óptimo.

Validado dicho instrumento, se aplicó el pretest para ambos grupos para conocer el nivel de motivación docente en el Instituto de Educación Superior Computron. Ello demostró que la motivación era baja en ambos casos.

Dos meses después de la culminación del experimento en diciembre del 2013, en febrero del 2014 se desarrolló el postest para efectuar las comparaciones del caso entre el grupo experimental y el grupo control, demostrando que los 33 docentes del Instituto de Educación Superior Computron de Los Olivos el evaron significativamente su nivel de motivación gracias al desarrollo del programa de gestión educativa con las técnicas de relaciones públicas; en cambio, los otros 35 docentes del grupo control mantuvieron sus niveles bajos de motivación al no haber sido sometidos al experimento, hecho que demostró la validez del programa.

Según el método desarrollado, el estudio de la presente tesis es de campo porque busca resolver el problema consistente en la poca motivación e identificación del personal docente respecto a la institución educativa para la cual labora, hecho que se puede ver en la realidad y específicamente en el Instituto de Educación Superior Computron en la ciudad de Lima. A su vez, siguiendo la clasificación que plantea Hernández, Baptista \& Fernández (1997, p. 321) la presente investigación es "explicativa, correlacional, longitudinal, prospectivo cuasiexperimental." 
Es explicativa porque descubre las causas de los bajos niveles de motivación del personal docente en cuanto al por qué en el instituto de Educación Superior Computron, dicho personal no tienen una identificación adecuada hacia la misma institución educativa a conciencia $y$, por consiguiente, su participación en distintos certámenes académicos y sociales se efectúa de manera limitada. Por ello se considera como hipótesis que la aplicación de las técnicas de relaciones públicas en un programa de gestión educativa que desarrollará la dirección administrativa del instituto de Educación Superior Computron soluciona eficientemente este problema logrando que su gestión educativa logre que el personal docente al estar motivado y plenamente identificado con la misma institución educativa se vea reflejado en una prestación de un servicio educativo de mayor calidad y que a su vez que las participaciones en diversas actividades sociales y académicas se basen en una convicción de pertenencia a la institución educativa y no por coerción, como en cierta forma se vino haciendo hasta el momento.

Es correlacional porque busca relacionar la influencia de la variable independiente " $X$ " que viene a ser la aplicación del programa de gestión educativa junto con la aplicación de las técnicas de relaciones públicas, sobre la variable dependiente " $y$ " que viene a ser el nivel de motivación docente en el Instituto de Educación Superior Computron del distrito de Los Olivos, en el cual se busca demostrar que la aplicación de la variable independiente es el factor crucial que influye positiva y significantemente sobre la variable dependiente.

Es longitudinal porque abarca un periodo de estudios de seis meses, desde la aprobación del proyecto de la presente investigación hasta la aplicación de los postests, razón por la que mide la evolución del mejoramiento del nivel de motivación de los docentes del Instituto de Educación Superior Computron de la sede del distrito de Los Olivos antes, durante y después de la aplicación del experimento, el cual consistió en la aplicación de las técnicas de relaciones públicas en los programas de gestión educativa que desarrolla la dirección ejecutiva de esta entidad de educación superior.

Es prospectivo porque los resultados obtenidos sirven para futuras investigaciones sobre la eficiencia de la gestión educativa en instituciones educativas de cualquier nivel, sea escolar, superior o universitaria, tomando como modelo la aplicación de las técnicas de relaciones públicas para el logro de dicho fin. El mejoramiento de los niveles de motivación de los docentes y por consiguiente la eficiencia de la gestión educativa en el Instituto de Educación Superior Computron hará posible que este programa piloto de gestión educativa se pueda generalizar y aplicar a todas las instituciones educativas, tanto de la ciudad de Lima, como del Perú y a nivel internacional, hecho que viene a ser la contribución de la presente investigación.

Por último, es cuasiexperimental porque ambos grupos no fueron seleccionados al azar. Y a estaban constituidos antes, durante y después de la experimentación.

\section{Resultados}

Se aplicó una metodología teórico-prácticodemostrativo-participativo solo al grupo denominado grupo experimental. Para ello, se aplicó un conjunto de técnicas de relaciones públicas durante el desarrollo del programa de gestión educativa, siendo la más aceptada la técnica de la administración de la controversia pública que logró tomar acuerdos mutuos entre los docentes y la dirección del Instituto de Educación Superior Computron representado por su gerente general, Lic. Richar B acón Terrones.

En el caso del grupo control, se observa que los resultados del pretest (entrada) y postest (salida) no tuvieron mayores cambios manteniéndose en el intervalo de $[17,32]$ del baremo, lo cual representa un nivel bajo de motivación docente según la tabla 9. Especificando este resultado, en el análisis de las cuatro dimensiones de motivación docente estudiadas en la presente investigación, se observa según las tablas 1, 2, 3 y 4 que los sujetos observados respondieron entre las escalas de frecuencias nunca, rara vez, a veces y regularmente sentirse motivados.

En el caso del grupo experimental, a quienes se les aplicó el programa de gestión educativa con las técnicas de relaciones públicas, se observa que los resultados del pretest (entrada) y postest (salida) sí tuvieron cambios significativos entre ellos, demostrando que el nivel de motivación docente de encontrarse en el intervalo $[17,32]$ del baremo, lo cual representa un nivel bajo de motivación antes del experimento, pasó a encontrarse en los intervalos $[49,64]$ y $[65,80]$, los cuales son superiores al baremo, hecho que lo ubica en los niveles alto y óptimo de motivación docente después de la aplicación del experimento, según la tabla 10. Especificando este resultado, en el análisis de las cuatro 
dimensiones de motivación docente estudiadas en la presente investigación, se observa que según las tablas 5 , 6,7 y 8 que los sujetos sometidos al programa de gestión educativa con las técnicas de relaciones públicas respondieron entre las escalas de frecuencias casi siempre y siempre sentirse motivados.

Los resultados de pretest (entrada) y postest (salida), tanto para el grupo control como para el grupo experimental, nos permiten comprobar en términos porcentuales las diferencias que surgieron en el nivel de motivación docente en ambos grupos durante la observación en el caso del grupo control (35 docentes del Instituto de Educación Superior Computron de San Juan de Lurigancho), y en el caso del grupo experimental antes del experimento y después de la aplicación del programa de gestión educativa con las técnicas de relaciones públicas (33 docentes del Instituto de Educación Superior Computron del distrito de LOS Olivos), obteniéndose las siguientes cifras:

Tabla 1.E stadísticos de la dimensión: realización personal de los docentes (grupo control)

\begin{tabular}{llcccccc}
\hline & & \multicolumn{3}{c}{ Grupo control entrada } & \multicolumn{3}{c}{ Grupo control salida } \\
\cline { 2 - 8 } & & $\mathbf{f}$ & $\%$ & $\%$ acum. & f & $\%$ & \% acum. \\
\hline Válido & Rara vez & 5 & 14,3 & 14,3 & 2 & 5,7 & 5,7 \\
& A veces & 19 & 54,3 & 68,6 & 23 & 65,7 & 71,4 \\
& Regularmente & 11 & 31,4 & & 10 & 28,6 & \\
& Casi siempre & & & & & & \\
& Siempre & & & & & & 100,0 \\
& Total & 35 & 100,0 & 100,0 & 35 & 100,0 & \\
\end{tabular}

Tabla 2. Estadísticos de la dimensión: reconocimiento de aportes de los docentes del grupo de control

\begin{tabular}{llcccccc}
\hline & & \multicolumn{3}{c}{ Grupo control entrada } & \multicolumn{3}{c}{ Grupo control salida } \\
\cline { 2 - 8 } & & $\mathbf{f}$ & $\%$ & $\%$ acum. & $\mathbf{f}$ & $\%$ & $\%$ acum. \\
\hline Válido & Nunca & 13 & 37,1 & 37,1 & 11 & 31,4 & 31,4 \\
& Rara vez & 6 & 17,1 & 54,3 & 3 & 8,6 & 40,0 \\
& A veces & 16 & 45,7 & 100,0 & 18 & 51,4 & 91,4 \\
& Regularmente & & & & 3 & 8,6 & 100,0 \\
& Total & 35 & 100,0 & & 35 & 100,0 & \\
\hline
\end{tabular}

Tabla 3. Estadísticos de la dimensión: responsabilidad docente de los profesores

\begin{tabular}{llcccccc}
\hline & & \multicolumn{3}{c}{ Grupo control entrada } & \multicolumn{3}{c}{ Grupo control salida } \\
\cline { 3 - 7 } & & $\mathbf{f}$ & $\%$ & $\%$ acum. & $\mathbf{f}$ & $\%$ & $\%$ acum. \\
\hline Válido & Rara vez & 1 & 2,9 & 2,9 & 3 & 8,6 & 8,6 \\
& A veces & 28 & 80,0 & 82,9 & 27 & 77,1 & 85,7 \\
& Regularmente & 6 & 17,1 & 100,0 & 5 & 14,3 & 100,0 \\
& Total & 35 & 100,0 & & 35 & 100,0 & \\
\hline
\end{tabular}

Tabla 4. Estadísticos de la dimensión: adecuación a las condiciones de trabajo de los docentes

\begin{tabular}{llcccccc}
\hline & & \multicolumn{3}{c}{ Grupo control entrada } & \multicolumn{2}{c}{ Grupo control salida } \\
\cline { 2 - 7 } & R & \% & \% acum. & f & $\%$ & $\%$ acum. \\
\hline Válido & 16 & 45,7 & 45,7 & 8 & 22,9 & 22,9 \\
& A veces & 19 & 54,3 & 100,0 & 26 & 74,3 & 97,1 \\
& Regularmente & & & & 1 & 2,9 & 100,0 \\
& Total & 35 & 100,0 & & 35 & 100,0 & \\
\hline
\end{tabular}


Como se puede observar en las tablas 1, 2, 3 y 4, los 35 docentes del grupo control correspondientes a la sede del distrito de San Juan de Lurigancho del Instituto de Educación Superior Computron manifestaron que nunca, rara vez, a veces y en pocos casos regularmente se sentían motivados, tanto antes (entrada) como después (salida) de la observación desarrollada en la presente investigación.

\section{Resultados del grupo experimental:}

Tabla 5. Estadísticos de la dimensión: nivel de realización personal de los docentes

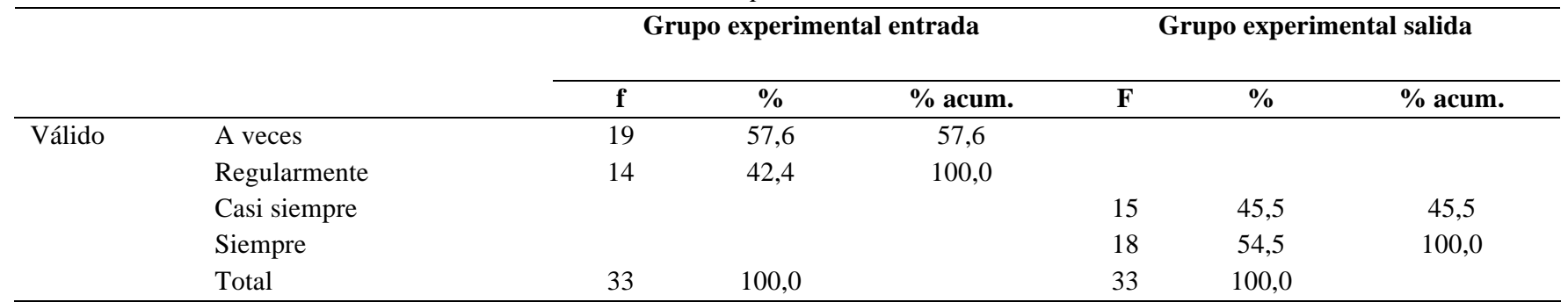

Tabla 6. Estadísticos de la dimensión: reconocimiento de aportes de los docentes

\section{G rupo experimental entrada G G $\quad$ rupo experimental salida}

\begin{tabular}{llcccccc} 
& & $\mathbf{f}$ & $\%$ & $\%$ acum. & $\mathbf{F}$ & $\%$ & \% acum. \\
\hline Válido & Nunca & 6 & 18,2 & 18,2 & & & \\
& Rara vez & 14 & 42,4 & 60,6 & & & \\
A veces & 12 & 36,4 & 97,0 & & & \\
Regularmente & & & & & & 27,3 \\
Casi siempre & & & & 9 & 27,3 & 100,0 \\
Siempre & 1 & 3,0 & 100,0 & 24 & 72,7 & \\
Total & 33 & 100,0 & & 33 & 100,0 & \\
\hline
\end{tabular}

Tabla 7. Estadísticos de la dimensión: nivel de responsabilidad docente de los profesores

\section{Grupo experimental entrada $\quad$ Grupo experimental salida}

\begin{tabular}{llcccccc} 
& & $\mathbf{f}$ & $\%$ & \% acum. & f & \% & \% acum. \\
\hline Válido & Rara vez & 2 & 6,1 & 6,1 & & & \\
& A veces & 20 & 60,6 & 66,7 & & & \\
& Regularmente & 11 & 33,3 & 100,0 & & & 36,4 \\
& Casi siempre & & & & 12 & 36,4 & 100,0 \\
& Siempre & & & & 21 & 63,6 & \\
& Total & 33 & 100,0 & & 33 & 100,0 & \\
\hline
\end{tabular}

Tabla 8. Estadísticos de la dimensión: nivel de adecuación a las condiciones de trabajo de los docentes

G rupo experimental entrada

Grupo experimental salida

\begin{tabular}{|c|c|c|c|c|c|c|c|}
\hline & & $f$ & $\%$ & $\%$ acum. & $f$ & $\%$ & $\%$ acum. \\
\hline \multirow[t]{6}{*}{ Válido } & Rara vez & 23 & 69,7 & 69,7 & & & \\
\hline & $A$ veces & 10 & 30,3 & 100,0 & & & \\
\hline & R egularmente & & & & & & \\
\hline & Casi siempre & & & & 12 & 36,4 & 36,4 \\
\hline & Siempre & & & & 21 & 63,6 & 100,0 \\
\hline & Total & 33 & 100,0 & & 33 & 100,0 & \\
\hline
\end{tabular}


aplicación del programa de gestión educativa con las técnicas de relaciones públicas manifestaron que nunca, rara vez, a veces y en pocos casos regularmente se sentían motivados. Esta situación cambió significativamente después (datos de salida) de la aplicación del mencionado programa, lo cual se puede observar cuando manifestaron que casi siempre y siempre se sentían motivados, hecho que compureba la eficiencia de las técnicas de relaciones públicas en el mejoramiento del nivel de motivación docente.

\section{Comparación de resultados finales de ambos grupos}

Tabla 9. Edad *niveles de motivación de los docentes de ambos grupos, salida

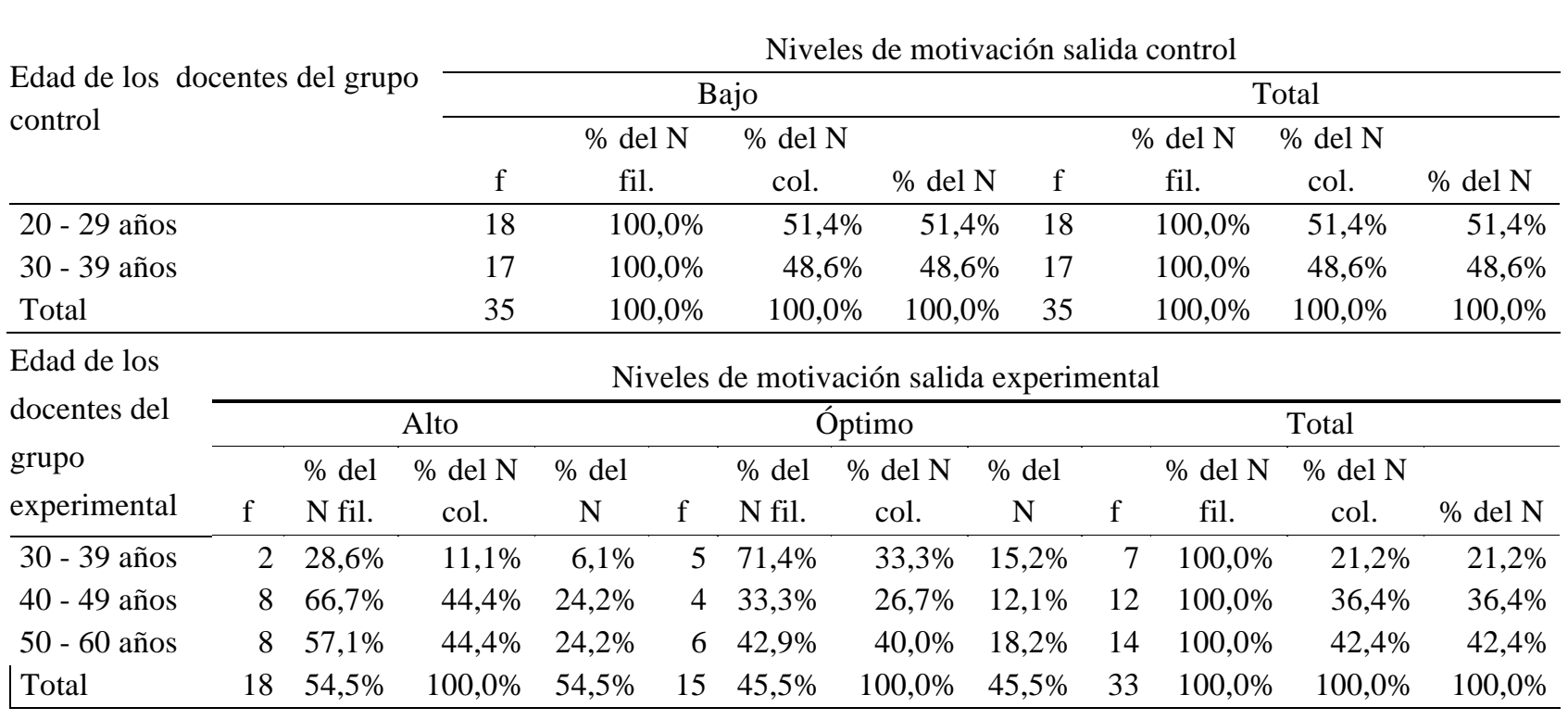

De la tabla 9 se puede observar, al término de la aplicación de las técnicas de relaciones públicas, que el grupo control se encuentra en el nivel bajo de motivación con puntajes comprendidos en el intervalo [17,32]; en cambio, los docentes del grupo experimental se encuentran en los niveles superiores del baremo, alto $u$ óptimo, que corresponden respectivamente a los intervalos $[49,64]$ y $[65,80]$. Para el grupo control, el $51,4 \%$ de docentes pertenecen al nivel de motivación bajo y tienen una edad comprendida en el intervalo cerrado [20, 29]; para el grupo experimental: 1) el 24,2\% de docentes pertenecen al nivel de motivación alto y tienen una edad comprendida en el intervalo [40, 49], e igualmente el $24,2 \%$ de docentes pertenecen al nivel de motivación alto y tienen una edad comprendida en el intervalo $[50,60], 2)$ el $18,2 \%$ de docentes pertenecen al nivel de motivación óptimo y tienen una edad comprendida en el intervalo cerrado [50,60]. Al término de la aplicación de las técnicas de relaciones públicas, el grupo control se mantiene en el mismo nivel de motivación, bajo; en cambio, el grupo experimental se ubica en las dos últimas categorías del extremo derecho del baremo, alto u óptimo. Esto corrobora la efectividad de la aplicación de dichas técnicas. 
Tabla 10. Género *niveles de motivación de los docentes de ambos grupos, salida

\begin{tabular}{|c|c|c|c|c|c|c|c|c|}
\hline \multirow{3}{*}{$\begin{array}{l}\text { Género de los docentes- } \\
\text { del grupo control }\end{array}$} & \multicolumn{8}{|c|}{ Niveles de motivación salida control } \\
\hline & \multicolumn{4}{|c|}{ Bajo } & \multicolumn{4}{|c|}{ Total } \\
\hline & $f$ & $\begin{array}{l}\text { \% del N de } \\
\text { fila }\end{array}$ & $\begin{array}{l}\% \text { del } \mathrm{N} \text { de } \\
\text { columna }\end{array}$ & $\begin{array}{c}\% \text { del N de } \\
\text { tabla }\end{array}$ & $f$ & $\begin{array}{l}\% \text { del } N \text { de } \\
\text { fila }\end{array}$ & $\begin{array}{l}\% \text { del } \mathrm{N} \text { de } \\
\text { columna }\end{array}$ & $\begin{array}{c}\% \text { del } \mathrm{N} \text { de } \\
\text { tabla }\end{array}$ \\
\hline M asculino & 17 & $100,0 \%$ & $48,6 \%$ & $48,6 \%$ & 17 & $100,0 \%$ & $48,6 \%$ & $48,6 \%$ \\
\hline Femenino & 18 & $100,0 \%$ & $51,4 \%$ & $51,4 \%$ & 18 & $100,0 \%$ & $51,4 \%$ & $51,4 \%$ \\
\hline Total & 35 & $100,0 \%$ & $100,0 \%$ & $100,0 \%$ & 35 & $100,0 \%$ & $100,0 \%$ & $100,0 \%$ \\
\hline
\end{tabular}

\begin{tabular}{|c|c|c|c|c|c|c|c|c|c|c|c|c|}
\hline \multirow{3}{*}{$\begin{array}{l}\text { Género de los docentes } \\
\text { del grupo experimental }\end{array}$} & \multicolumn{12}{|c|}{ Niveles de motivación salida experimental } \\
\hline & \multicolumn{4}{|c|}{ Alto } & \multicolumn{4}{|c|}{ Optimo } & \multicolumn{4}{|c|}{ Total } \\
\hline & f & $\begin{array}{l}\% \text { del } \\
\mathrm{N} \text { fil. }\end{array}$ & $\begin{array}{l}\% \text { del N } \\
\text { col. }\end{array}$ & $\%$ del $\mathrm{N}$ & f & $\begin{array}{l}\% \text { del } \\
\mathrm{N} \text { fil. }\end{array}$ & $\begin{array}{l}\% \text { del N } \\
\text { col. }\end{array}$ & $\begin{array}{c}\% \text { del } \\
N\end{array}$ & $f$ & $\begin{array}{l}\% \text { del } \mathrm{N} \\
\text { fil. }\end{array}$ & $\begin{array}{c}\% \text { del } \mathrm{N} \\
\text { col. }\end{array}$ & $\begin{array}{c}\% \text { del } \\
\mathrm{N}\end{array}$ \\
\hline M asculino & 8 & $50,0 \%$ & $44,4 \%$ & $24,2 \%$ & 8 & $50,0 \%$ & $53,3 \%$ & $24,2 \%$ & 16 & $100,0 \%$ & $48,5 \%$ & $48,5 \%$ \\
\hline Femenino & 10 & $58,8 \%$ & $55,6 \%$ & $30,3 \%$ & 7 & $41,2 \%$ & $46,7 \%$ & $21,2 \%$ & 17 & $100,0 \%$ & $51,5 \%$ & $51,5 \%$ \\
\hline Total & 18 & $54,5 \%$ & $100,0 \%$ & $54,5 \%$ & 15 & $45,5 \%$ & $100,0 \%$ & $45,5 \%$ & 33 & $100,0 \%$ & $100,0 \%$ & $100,0 \%$ \\
\hline
\end{tabular}

De la tabla 8 se puede observar, al término de la aplicación de las técnicas de relaciones públicas, que el grupo control se encuentra en el nivel bajo de motivación con puntajes comprendidos en el intervalo [17,32]; en cambio, los docentes del grupo experimental se encuentran en los niveles superiores del baremo, alto $u$ óptimo, que corresponden respectivamente a los intervalos $[49,64]$ y $[65,80]$. Para el grupo control, el $51,4 \%$ de docentes tiene un nivel de motivación bajo y pertenece al género femenino; para el grupo experimental, el $30,3 \%$ de docentes pertenecen al nivel de motivación alto y al género femenino. Al término de la aplicación de las técnicas de relaciones públicas, para el grupo control, en el nivel óptimo presentan mayor motivación los hombres que las mujeres; en el nivel alto, hay mayor motivación de las mujeres que los hombres.

En general, en el grupo experimental el $54,5 \%$ se encuentra en el nivel al to y el $45,5 \%$ en el nivel óptimo. $\mathrm{Si}$ nos referimos al estudio de la motivación, el antecedente principal de su estudio viene a ser M aslow, quien es citado por Calderón (1996, p. 24) quien establece una escala de siete motivaciones y que el cumplimiento de una era requisito para que se vayan dando las siguientes. Sin embargo, afirma que "para el trabajo de las organizaciones estas fueron reducidas a cinco, siendo las siguientes: Necesidad fisiológica, de seguridad, de afiliación o pertenencia social, de reconocimiento social y el de la autorrealización".

En la presente investigación se considera dentro de las dimensiones de estudio de la motivación en los problemas, hipótesis y objetivos específicos objetos de nuestra observación y aplicación de soluciones a tres de las cinco necesidades que nos expone Maslow: la realización personal propiamente dicha, el reconocimiento de sus aportes (reconocimiento social), desarrollo de la responsabilidad (de afiliación 0 pertenencia social) y el de la adecuación al ambiente de trabajo (necesidad fisiológica). Esta teoría está graficada por la pirámide de las necesidades humanas de M aslow.

Gráfico 1: Las necesidades según la teoría de M aslow en el desempeño laboral (Amaru M. 2008).

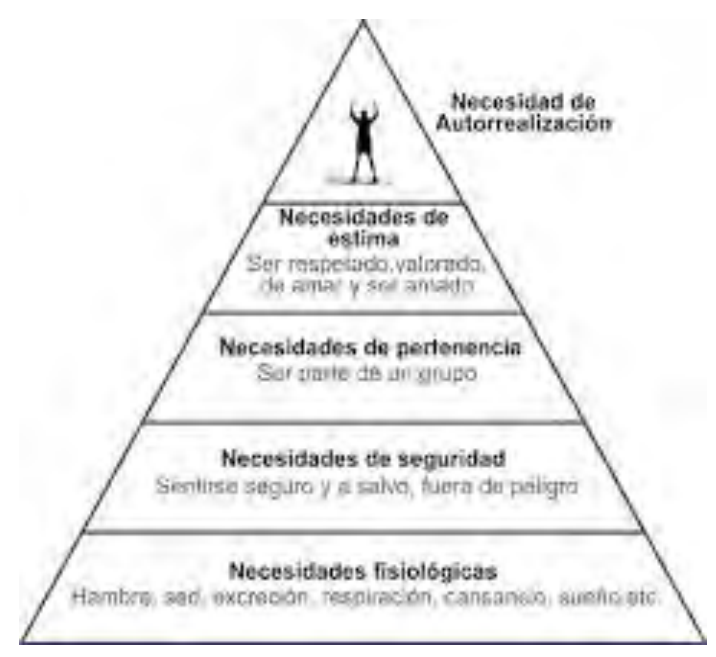

Una de las necesidades más importantes para el desarrollo de la motivación del personal de una organización, en este caso de los docentes, es el de la 
optimización de la comunicación interna en cualquier tipo de organización y con mayor razón si dicha institución es educativa en donde se debe predicar con el ejemplo mediante una relación basada en la confianza y los valores entre directivos y docentes.

Y a lo hizo notar hace una década atrás el tratadista de las relaciones públicas Seitel $(2002$, p. 51) cuando sostuvo que "la práctica de la comunicación interna implica la planificación de esta, a partir de una investigación en la institución para conocer las necesidades de su público interno y los instrumentos que determinarán sus políticas y objetivos organizacionales". Es decir que dicha situación ideal es generada por un trabajo de relaciones públicas enmarcadas en el desarrollo de políticas que hayan sido aceptadas y aprobadas en conjunto con el personal que conforma dicha organización, por lo que, trasladando esta afirmación hacia el caso de las instituciones educativas, la gestión que desarrollen los directivos de estos centros deben estar pendientes de las necesidades y expectativas de sus propios docentes, hecho que no es un trabajo aislado sino planificado en donde la participación de todos los maestros es prioritaria y que a su vez esta redundará en la formación de un buen nivel de motivación docente basado en la buena comunicación.

A ello K ovács (2007, p. 126) completa esta afirmación cuando replantea la máxima de las relaciones públicas cuando indica que "Las organizaciones mediante el ejercicio de las relaciones públicas deben hacer las cosas bien, hacerlas saber y hacerlas sentir". El hacer sentir completa el resultado de una comunicación participativa entre todos los miembros de una organización determinada, ya que si a los públicos internos se les permite participar desde la planificación en distintas tomas de decisiones, ello generará que dicho personal sienta como propio cualquier logro que se obtenga gracia a este esfuerzo conjunto.

A ello se añade además una necesidad que $M$ aslow hizo notar tomando en cuenta la natural eza social del hombre: la del reconocimiento. Vale decir aquí que a cualquier colaborador de una organización a quien se le tome en cuenta para la toma de decisiones en una gestión, le causará una motivación significativa de querer pertenecer y dar lo mejor de sí a dicha institución.

Siendo la motivación, una de las variables de estudio, en este caso del personal docente del Instituto de Educación Superior Computron, y a su vez complementando los fundamentos teóricos expuestos por M aslow, también se considera a los factores que favorecen dicha motivación por parte de los colaboradores hacia una organización. Esto es coincidentemente expuesto por Papalia \& W endkos (1993, p. 323) quienes afirman que "M aslow propugnó que una de las metas más ansiadas por el ser humano es el encontrar la satisfacción por la realización de su propio potencial". Es decir, que uno de los factores más importantes para el hombre es la autorrealización personal, hecho en el cual interviene en forma decisiva la gestión que la dirección puede desarrollar en una organización a favor de sus colaboradores. Esta situación puede ser facilitada con una buena comunicación en donde exista respeto, confianza y al mismo tiempo que los directivos de una organización cualquiera puedan fomentar la participación activa en los quehaceres de la organización a sus colaboradores. Cuando el colaborador siente que es tomado en cuenta, entonces se identifica y se motiva en dar lo mejor de sí a favor de la organización misma, y mejor aún si esta participación tiene alguna compensación social y moral que también, a decir de M aslow, es importante para mejorar el potencial del ser humano y por ende un factor decisivo para su motivación.

La relación de la comunicación con el desarrollo del potencial humano también es citada por Solórzano (1995, p. 28) cuando sostiene que "Es aquí donde las relaciones públicas asumen nuevos roles. Por ello es que en el pensamiento y en la acción de los relacionistas debe estar presente la integración, entendida esta como el camino que contribuya al logro de la realización del ser humano". Comprendida esta relación, es que en el presente trabajo se buscará demostrar que en la problemática de la motivación docente en el instituto de Educación Superior Computron, un nuevo rol puede cumplir el desarrollo de una gestión educativa coherente con la aplicación de las técnicas de relaciones públicas, favorecerá la buena comunicación entre directivos y docentes y al mismo tiempo serán estos factores que motivarán al personal docente a colaborar decididamente con el engrandecimiento de dicha institución educativa, hecho que servirá de modelo para ser aplicada a cualquier clase de institución educativa en el país y en el mundo. Esta será la contribución al conocimiento de la gestión educativa. 


\section{Condusión}

La aplicación de un programa de gestión educativa resultó ser más eficiente cuando se desarrolló junto con las técnicas de relaciones públicas para mejorar el nivel de motivación docente en el Instituto de Educación Superior Computron del distrito de Los Olivos, en tanto que a falta de la aplicación de este programa no mejoró el nivel de motivación docente en la sede del distrito de San J uan de L urigancho de la misma institución.

Por ello la presente investigación nos enseña que es importante la comunicación permanente entre directivos y docentes para tomar decisiones concertadas a pesar de la existencia de diversidad de ideas u opiniones, que si no son tratadas a tiempo, podrían generar conflictos y un clima laboral negativo para el desarrollo de la institución educativa en sí. El logro de este fin se da gracias a la aplicación de la técnica de relaciones públicas de la controversia pública, por lo que se recomienda su aplicación en toda clase de institución educativa para mantener en alto el nivel de motivación docente, tomando en cuenta que es este recurso humano que prestigia en gran medida a la institución, hecho que contribuye al éxito de una gestión educativa.

\section{Referencias}

A maru M aximiliano, A ntonio C. (2008). Administración para emprendedores: fundamentos para la creación y gestión de nuevos negocios. Editorial Pearson Educación. M éxico D.F. (p.124).

Calderón Quino, Fernando. (1996). Relaciones Públicas: Calidad total y el factor humano. Editorial de la Escuela profesional de ciencias de la Comunicación de la U niversidad de San M artín de Porres. Lima Perú. Primera edición. (p. 24).

Hernández Sampieri, Roberto; Fernández Collado, Carlos \& Baptista Lucio, Pilar. (1997). Metodología de la investigación. Editorial MC Graw Hill. Bogotá - Colombia. (p.321).

Knight, George R. (2002). Filosofía y educación. Una introducción en la perspectiva cristiana. Asociación publicadora interamericana. Grupo OP. Colombia. Primera impresión. (p. 176).

Kovács Halay, István J. (2007). Relaciones Públicas: Ética, valores y teorías. Editorial Universidad Peruana Unión. Facultad de Ciencias Humanas y Educación. Escuela Académico Profesional de Ciencias de la Comunicación. Lima - Perú. ( $p$. 126).
Papalia, Diane \&Wendkos, Sally (1993) Psicología. Editorial M c Graw Hill. M éxico D.F. (p. 323).

Pereyra, Mario (2006). Psicología positiva. Editorial Universidad Peruana Unión. Primera edición. ( $p$. 71)

Ríos Szalay, Jorge (1994). Relaciones Públicas: Su administración en las organizaciones. Editorial Trillas. Segunda edición. M éxico D.F. - M éxico. (p. 37)

Sander Espinoza, B enno (2005). Nuevas tendencias en la gestión educativa. Tesis de licenciatura. Universidad A utónoma de M éxico. M éxico.

Seitel, Fraser (2002). Técnicas de Relaciones Públicas. Editorial Limusa. M éxico D.F. (p.51)

Solórzano Hernández, Emilio. (1995). Colección de autores. 10 FORO IBEROAMERICANO DE RELACIONES PÚBLICAS. Madrid - España junio de 1995. Ediciones Facultad de Ciencias de la Comunicación. Universidad de San Martín de Porres. Lima - Perú (p.28) 